\title{
Epistemologías y metodologías críticas en Ciencias Sociales: precisiones conceptuales en clave latinoamericana
}

\author{
Critical epistemologies and methodologies in Social Sciences: conceptual \\ clarifications from a Latin American perspective
}

\author{
María Mercedes Palumbo \\ mer.palumbo@gmail.com \\ Universidad Nacional de Luján-CONICET/ \\ Universidad de Buenos Aires (UBA), Argentina \\ Laura Celina Vacca \\ celinavacca@yahoo.com.ar \\ Universidad Nacional de General San Martín \\ (UNSAM)/Universidad de Buenos Aires (UBA), \\ Argentina
}

Cita sugerida: Palumbo, M. M. y Vacca, L. C. (2020).

Epistemologías y metodologías críticas en Ciencias

Sociales: precisiones conceptuales en clave

latinoamericana. Revista Latinoamericana de

Metodología de las Ciencias Sociales, 10(2), e076.

https://doi.org/10.24215/18537863e076

Recepción: 04 Abril 2020

Aprobación: 06 Junio 2020

Publicación: 01 Diciembre 2020

\begin{abstract}
Resumen: El objetivo general del artículo consiste en revisitar cuatro perspectivas epistemológicas y metodológicas críticas en el campo de las Ciencias Sociales. Particularmente, se retoman la investigación-acción participativa, la investigación militante, la investigación en colaboración y las metodologías descoloniales o descolonizadoras. Desde una metodología hermenéuticointerpretativa, se analizan sus acercamientos fundados en las críticas compartidas al positivismo y su común tradición latinoamericana. Asimismo, se aborda un aspecto menos indagado, relativo a la existencia de una serie de matices entre las perspectivas, que permiten resaltar la singularidad de cada una. Las conclusiones sostienen la importancia de considerar la dimensión metodológica para concretizar los puntos de partida epistemológicos.
\end{abstract}

Palabras clave: Epistemología, Metodología, Crítica, Ciencias Sociales, América Latina.

\begin{abstract}
The general aim of this article is to revisit four critical epistemological and methodological perspectives in the research field of Social Sciences. Specifically, the following perspectives have been selected: participatory action research, militant research, collaborative research and decolonial or decolonizing methodology. From a hermeneutic-interpretive methodology their similarities are analyzed with respect to their critics to positivism and their common Latin American tradition. Moreover, it addresses a less examined aspect related to a series of nuances among perspectives, that allow the underlining of the singularity of each one. The conclusions stress the importance of taking into account the methodology dimension in order to concretize the epistemological startpoints.
\end{abstract}

Keywords: Epistemology, Methodology, Critic, Social Science, Latin America.

\section{INTRODUCCIÓN}

El presente artículo toma como punto de partida la inquietud por el lugar que ocupan las perspectivas epistemológicas y metodológicas críticas en la investigación en el campo de las Ciencias Sociales. ${ }^{1}$ Particularmente, se busca establecer un diálogo con una tradición latinoamericana ${ }^{2}$ que reflexiona sobre las 
prácticas científicas a la hora de producir conocimiento en movimientos sociales y populares, desde ellos y sobre ellos (Fajardo, Longa y Stratta, 2012).

Si bien los cuestionamientos a la epistemología tradicional de corte positivista se encuentran extendidos en las academias, sostenemos a modo de hipótesis interpretativa que existe una cierta indistinción terminológica al interior de las perspectivas críticas en tanto se utilizan denominaciones disímiles para dar cuenta de abordajes similares, o bien, por el contrario, se engloban prácticas investigativas diferentes bajo un mismo nombre. Adicionalmente, entendemos que en las perspectivas críticas se encuentra un fuerte desarrollo epistemológico, traccionado por la búsqueda de ruptura del canon científico, que no se corresponde con igual desarrollo en lo referente a los abordajes metodológicos. Justamente es en el campo de la metodología donde, a menudo, se despliegan una serie de tensiones y desafíos en el diálogo entre actores y lógicas universitarios y extrauniversitarios.

Si consideramos la inquietud de partida y las hipótesis interpretativas mencionadas, el objetivo general del artículo consiste en revisitar cuatro perspectivas epistemológicas y metodológicas críticas. Se seleccionaron la Investigación-Acción Participativa (IAP), la Investigación Militante (IM), la Investigación en Colaboración (IeC) y las Metodologías Descoloniales o Descolonizadoras (MD) como perspectivas a analizar. Su selección se basa en su importancia a la hora de pensar modos de hacer ciencia crítica latinoamericana; esto es, una ciencia que se posiciona desde una relación de compromiso (Elías, 1990) con los objetos/sujetos de estudio en América Latina y desde América Latina. Desde una metodología hermenéutico-interpretativa nos interesa tanto describir sus acercamientos fundados en las críticas compartidas al canon positivista y su común inscripción latinoamericana, como también avanzar en el análisis de una serie no menor de matices que permiten recuperar la singularidad de cada una de ellas.

Para ello, la presentación de las reflexiones inicia con una breve caracterización de las cuatro perspectivas elegidas, considerando la tradición latinoamericana que las aúna y la relevancia de Orlando Fals Borda como referente reiteradamente recuperado. A continuación, se ponen en diálogo dichas perspectivas a partir de establecer tres elementos compartidos: a) el esquema sujeto-sujeto frente a una ciencia erigida sobre la relación sujeto-objeto; b) la vinculación del conocimiento científico con los saberes populares frente a una ciencia basada en la jerarquía y universalidad del primero; c) el compromiso de la ciencia con la política en contraposición a una ciencia desinteresada. Estos elementos compartidos son indagados y se hacen visibles los matices que se presentan en cada perspectiva, en un ejercicio de reflexividad pendiente en las Ciencias Sociales tendiente a rescatar la singularidad de cada planteo. Finalmente, se prosigue con la recapitulación de los principales argumentos esbozados y la recuperación de los desafíos metodológicos que interpelan a los investigadores que se inscriben en estas perspectivas críticas.

\section{Perspectivas epistemológicas y metodológicas críticas en clave LATINOAMERICANA}

Los debates y aportes en torno a la epistemología y metodología tienen una larga y amplia tradición alimentada por perspectivas muy diversas con respecto a sus posicionamientos en torno a la producción de conocimiento. Aquí hacemos hincapié en una discusión renovada en las últimas décadas en el campo de las Ciencias Sociales en América Latina, a partir de un conjunto de textos de bibliografía especializada orientada a reflexionar sobre prácticas de investigación que plantean una relación de coproducción de conocimiento junto con los sujetos de estudio. Esta discusión la encontramos sistematizada principalmente -aunque no exclusivamente $-{ }^{3}$ en cuatro perspectivas principales: la IAP, la IM, la IeC y las MD.

A pesar de que el objetivo de este artículo es avanzar hacia la singularización de estas perspectivas, nos interesa puntualizar en la existencia de una tradición común a las mismas, que explica el amplio corpus de puntos de acuerdo. Más aún, hallamos una genealogía propiamente latinoamericana ${ }^{4}$ que conduce a la figura de Orlando Fals Borda y, de modo secundario aunque igualmente destacado, a Paulo Freire como referencias 
fundacionales de un modo latinoamericano de hacer ciencia. Es posible destacar, tal como lo plantea Michi (2020), tres aspectos que vuelven a estos autores un mojón en la ciencia latinoamericana crítica de corte antipositivista: por un lado, la apuesta por aunar producción de conocimiento y acción política en el estudio de una realidad concreta desde el compromiso con la transformación social insertando la investigación en una práctica colectiva; por otro, la vinculación de los sujetos de la ciencia con los "sujetos de a pie" en un diálogo entre conocimientos diferentes pero igualmente incompletos, y finalmente, el reconocimiento del proceso educativo que media esta forma de hacer ciencia en la que transitan aprendizajes todos los sujetos involucrados.

En este marco, la IAP se origina en las décadas del sesenta y setenta, y el Simposio Mundial de Investigación Acción en Cartagena de 1977 resultó un hito fundamental para su consolidación. La impronta de Fals Borda emerge como perspectiva epistemológica y metodológica, a la vez que antecedente fundante de las otras perspectivas aquí revisitadas. Si seguimos a Herrera Farfán, "La IAP es una apuesta teórico-política, donde las redefiniciones conceptuales tienen implicaciones políticas en función de las luchas y las necesidades de las comunidades organizadas" (2018, p. 78). Es decir, el horizonte y el ordenador de la investigación, y el rol de los investigadores, es la transformación social. Esto ha tenido distintos ajustes y relecturas a lo largo del tiempo, de las que una de las principales es la elaboración de Sirvent y Rigal (2012, 2014). Junto con el objetivo político de emancipación y construcción de subjetividades críticas, se busca que el conocimiento sea coproducido entre los investigadores y los sectores populares involucrados en el proceso, a partir de una relación dialógica que recupere la experiencia práctica de estos actores.

En cuanto a la IM, seguimos la línea de interpretación de Michi (2020), quien la sitúa como una refundación política contemporánea de la IAP; refundación, en cuanto coloca la idea de compromiso político con la transformación social en el centro de la escena, amplía las problemáticas y formas de investigación a todas aquellas que resulten necesarias para la praxis cotidiana de las organizaciones y movimientos populares, y ubica a los protagonistas de las luchas como sujetos investigadores (Bringel y Versiani, 2016a, 2016b; Jaumont y Versiani, 2016; Bringel y Maldonado, 2016). El término investigación militante "busca designar ese amplio espacio de producción de conocimiento orientado a la acción transformadora que busca aliar la reflexión crítica y teórica en las luchas populares en un proceso multidireccional, articulando intelectuales, investigadores, movimientos sociales, comunidades y organizaciones políticas" (Jaumont y Versiani, 2016, p. $433)^{5}$. En suma, la noción de militancia sintetiza el compromiso ético y político con el cambio social, lo cual implica un fuerte anudamiento entre producción de conocimiento, acción política y cambio de la realidad social.

En el llamado giro decolonial o grupo Modernidad/Colonialidad se evidencia una apuesta epistemológica crítica, que discute las herencias coloniales de la producción científica en América Latina (Lander, 2000; Mignolo, 2010; Walsh, 2007). Principalmente, se cuestiona la subalternización de sujetos, saberes y prácticas en función a jerarquías étnico/raciales instauradas desde la conquista/invasión española. Esto ha sido estructural para la consolidación de un modelo científico hegemónico y eurocéntrico que parece ubicarse en un lugar de neutralidad. Desde esta epistemología crítica se aboga por un diálogo de saberes que recupere aquellos encarnados por los sujetos populares y que apunte a una descolonización del conocimiento (Castro Gómez, 2007). Estos autores han realizado importantes aportes en términos epistemológicos, mas no abordan en igual medida cuestiones metodológicas. No obstante, es posible encontrar producciones que se inscriben en este posicionamiento teórico-político, y se preguntan acerca de cómo poner en práctica metodologías descoloniales o descolonizadoras desde enfoques más situados (Borsani, 2014; Haber, 2011, 2014; Leyva y Speed, 2018; Guelman y Palumbo, 2018; Puentes, 2015; Segato, 2013; Smith, 2016). Así, la producción de una investigación descolonizadora está vinculada con la puesta en práctica de estrategias metodológicas diversas, permeables las demandas y problemas de los actores subalternos con los que se trabaja en la búsqueda por descolonizar el proceso de trabajo en su conjunto, más que los resultados en sí mismos. 
Por último, interesa destacar una serie de elaboraciones que reflexionan, mayormente desde el campo antropológico, sobre las relaciones de colaboración entre investigadores y sujetos de estudio. Siguiendo a Lassiter (2005), la noción de colaboración fue adquiriendo relevancia teórica hasta convertirse en un enfoque metodológico preciso. En estas producciones, la colaboración con los sujetos opera como principio explícito y guía para la investigación (Katzer y Samprón, 2012). El trabajo de campo deviene tarea de coteorización entre académicos y sujetos investigados. Se busca así que las interpretaciones de los procesos vividos por los actores emerjan de un diálogo reflexivo y colectivo, y "no de la interpretación académica del discurso y la práctica activista” (Rappaport, 2007, p. 221).

Consideramos que son tres los puntos de contacto centrales entre las cuatro perspectivas en indagación. En primer lugar, redefinen la relación sujeto-objeto a partir de plantear un esquema sujeto-sujeto que reconfigura los roles y grados de participación en la praxis investigativa. Un segundo punto refiere al quiebre de la supremacía del conocimiento científico, erigido sobre la jerarquización de la episteme sobre la doxa, con vistas a reconocer y recuperar la trama epistémica diversa de saberes ancestrales, colectivos y populares que convive con el conocimiento científico. En tercer lugar, rechazan la neutralidad valorativa y una ciencia desinteresada, que escinde a los juicios de hecho de los juicios de valor, dado su compromiso ético y político con los problemas sociales y con las luchas y realidades cotidianas, a las que se enfrentan las organizaciones y movimientos populares con los que los investigadores se vinculan en su práctica académica.

Cabe notar que las coincidencias existentes entre estas perspectivas operan en el sentido de cuestionar la ciencia moderna occidental de corte positivista en sus modos de hacer ciencia de lo social. Es el "pathos de la distancia” (Castro Gómez, 2007, p. 89) su marca distintiva o, en términos de Elías (1990), el "distanciamiento", que fija a los sujetos investigadores en la posición de observador de los fenómenos sociales como reaseguro de la objetividad del conocimiento producido. La novedad que traen estos planteos críticos radica en explorar el compromiso como posibilidad alternativa al distanciamiento, exploración que sitúa a los sujetos frente a potencialidades de enriquecimiento, tanto como a desafíos en la praxis investigativa concreta.

\section{LOS MATICES ENTRE PERSPECTIVAS: LA SINGULARIDAD DENTRO DEL COMÚN GESTO CRÍ́TICo}

\subsection{El esquema epistémico sujeto-sujeto}

Resulta interesante partir de la consideración de Katzer y Samprón (2012) respecto a la existencia de dos abordajes oposicionales a la hora de comprender la relación sujeto-objeto, que alternativamente polarizan o bien idealizan la interacción entre los sujetos. En cuanto a la polarización, situamos a la epistemología tradicional y su mencionado pathos de la distancia, que concibe al otro como un "informante" proveedor de información, separando los marcos cognitivos y normativos de los sujetos conforme a la posición desempeñada (investigador/a o investigado/a). En el extremo opuesto, se sostiene la indistinción entre los participantes en su condición de sujetos investigadores, minimizando las consideraciones relativas a la asimetría de poder y la distribución desigual de capitales sociales, económicos y culturales existente entre los mismos.

Las perspectivas aquí revisitadas plantean un fuerte cuestionamiento al dualismo sujeto-objeto, a la mencionada polarización. A contramano, se postula un esquema epistémico sujeto-sujeto fundado en la constatación de la existencia de múltiples reflexividades en juego en el proceso de construcción de conocimiento que alienta la activa participación de los sujetos en el derrotero de la investigación. ${ }^{6}$ De este modo, se desobjetiviza a los sujetos estudiados, tanto como se reconoce al/a la investigador/a en su doble rol de observador/a y participante de la realidad social. El corrimiento en los modos de concebir a los sujetos 
genera mutaciones en la naturaleza de la actividad científica misma tornándola, al menos como axioma e intencionalidad de partida, más abierta, democrática y participativa.

Asimismo, consideramos que estas perspectivas buscan alejarse del extremo contrario propio de la idealización ingenua que aplana las complejidades inherentes a sostener el igual estatus de los sujetos en torno al vínculo epistémico. Aun cuando encontramos variados ejercicios de reflexividad acerca de los desafíos implicados en la relación sujeto-sujeto, consideramos que el riesgo de la idealización no debe ser descartado de plano en relación a estas perspectivas, dado que resulta una posibilidad más cierta frente al riesgo de la polarización.

Ahora bien, las nominaciones empleadas tanto para dar cuenta del estatus otorgado a ese otro devenido sujeto de la investigación, que ya no opera como informante, como para las reconfiguraciones del sujeto investigador/a y las instancias pensadas y practicadas de su participación, otorgan pistas para pensar los matices entre perspectivas.

En el caso de la IAP, la apuesta por un modelo democrático de construcción de conocimiento asume el desarrollo de una teorización colectiva en un "esquema sujeto-sujeto" (Fals Borda en Herrera Farfán y López Guzmán, 2012, p. 132). El deslizamiento del informante hacia el "sujeto reflexivo" (Sirvent y Rigal, 2014, p. 11) da cuenta de una participación activa no solo en la provisión de información -el rol clásico de "objeto explotable" (Fals Borda en Herrera Farfán y López Guzmán, 2012, p. 244)-, sino también en la elaboración significativa de la misma, en la producción y transmisión de conocimiento sobre la realidad cotidiana devenida objeto de estudio. Aun más, en la medida en que los sujetos participan de la investigación, se involucran en un proceso de aprendizaje con un fuerte cariz político-pedagógico. De este modo, investigación, participación y praxis educativa operan como un trípode indisociable que requiere necesariamente de sujetos reflexivos o, en palabras de Fals Borda, una "alianza de sujetos activos" (Herrera Farfán y López Guzmán, 2012, p. 80).

Consideramos que un rasgo distintivo de la IAP se desprende de concretizar los modos de garantizar la interacción con "sujetos reflexivos" por medio del arbitrio intencional de instancias específicas de participación. Lo anterior se verifica desde la construcción del objeto pasando por las devoluciones sistemáticas, hasta la evaluación conjunta de los resultados, todas ellas instancias necesarias y previstas de antemano en el diseño de investigación. Un concepto clave es el de participación real (y no simbólica) definida como "la incidencia de la mayoría de la población en las decisiones que afectan su vida cotidiana" (Sirvent y Rigal, 2014, p. 8).

Aun cuando encontramos en Fals Borda un énfasis en las ideas de "reciprocidad simétrica" y "horizontalidad de sujeto a sujeto", propias de un momento fundacional de una ciencia latinoamericana en disputa con la epistemología tradicional, participación real no equivale a indistinción de sujetos. El/la investigador/a ocupa un rol específico y diferencial de coordinador/a de grupo y educador/a popular.

En línea con la idea de la IM como refundación de la IAP en un nuevo contexto, la concepción de sujetos reflexivos y activos sigue siendo cierta para esta perspectiva tanto como la democratización del proceso de investigación (desde la elección del tema, las herramientas, las reflexiones y las acciones). Para la IM, "los movimientos sociales y las colectividades dejan de ser meros objetos de estudio a ser observados de manera distante y neutra y pasan a ser considerados sujetos productores de conocimiento legítimo y participantes activos en la construcción de saber científico" (Bringel y Versiani, 2016a, p. 8) ${ }^{7}$. Empero, encontramos en la idea de "sujetos activos, actuantes y pensantes" (Jaumont y Versiani, 2016; Bringel y Versiani, 2016b) el modo específico de nombrar a los protagonistas de las luchas sociales participando de la praxis investigativa. No resulta casual la introducción del término actuante en dicha nominación, que marca un matiz en relación a la conceptualización de la IAP. La IM sostiene fuertemente el compromiso ético-político con la transformación social, y refiere a los actores de la acción colectiva como sus interlocutores. Si bien esta impronta también atraviesa la IAP, aquí encontramos un mayor énfasis en la dimensión política, a la que remite la propia noción 
de actuar, que se traduce en sujetos epistémicos (pensantes y actuantes en la investigación), pero ante todo políticos (actuantes en la transformación de un estado de cosas).

Esta posición científico-política compete igualmente al/a investigador/a, quien observa y reflexiona, pero a quien también se le exige accionar en los lugares pesquisados, lo que refuerza la imbricación entre investigación y acción política, teoría y práctica, trabajo intelectual y manual, entre el científico y el político. Aquí encontramos una segunda especificidad de la IM, que recoloca al/a la investigador/a como "un/a compañero/a de lucha". Resulta interesante convocar la idea de "parcería" (Jaumont y Versiani, 2016) como el modo en que se define el diálogo crítico que media la relación sujeto-sujeto en la praxis investigativa, devenida igualmente praxis política. En la referida "articulación académico-militante" (Bringel y Versiani, 2016b, p. 482) existen roles diferenciales, aunque no jerárquicos. Valiéndose del acumulado histórico de la IAP y de la educación popular freireana, el/la investigador/a deviene, al igual que en la IAP, coordinador/a, en términos de socialización de instrumentos teóricos, técnicos y prácticos relativos al conocimiento científico y aporta una comprensión crítica de la realidad.

En coincidencia con la IAP y la IM, la IeC convierte el trabajo de campo en coconceptualización, distanciándose de la concepción del mismo como instancia de recolección de datos (Rappaport, 2007). Lo anterior requiere necesariamente un corrimiento respecto a la idea del "informante" para dar lugar a una serie de nominaciones que coinciden en la agenciación otorgada a los sujetos para la labor conceptual, de interpretación y hasta de escritura en la investigación: consultor (Lassiter, 2005), coteorizador (Rappaport y Ramos Pacho, 2005) y socio epistémico (Marcus, 2008).

Ahora bien, la colaboración no implica una "reciprocidad balanceada" entre los sujetos intervinientes, que los colocaría en situación de simetría a la hora de establecer las características y rumbos de la interacción (Katzer y Samprón, 2012). La inscripción disciplinaria de destacados referentes de esta línea en la antropología podría explicar el aporte que encontramos en términos de un recaudo metodológico frente al extremo de la idealización ingenua. Se reiteran las referencias a las tensiones que surgen en el propio proceso de producción de conocimiento a la hora la construcción de textos compartidos, más allá de los axiomas epistemológicos de partida.

Una diferencia que hallamos entre la IAP y la IeC refiere a los modos de hacer efectiva la relación sujetosujeto: mientras la primera establece ciertas instancias preferentes y necesarias de participación (las ya citadas devoluciones sistemáticas, por ejemplo), la segunda presenta al trabajo de campo en su conjunto como espacio-momento de colaboración entre los distintos participantes. Una segunda distancia de la IeC en relación a la IAP, y fundamentalmente a la IM, radica en el tipo de redefinición que opera sobre el propio sujeto investigador, quien en un caso resulta ser un "compañero de lucha" y en el otro un sujeto investigador comprometido pero lejos de la fusión científico-política.

\subsection{El diálogo entre conocimiento científico y saberes populares}

De acuerdo al planteo de Castro Gómez (2007), una de las características principales a la episteme moderna colonial reside en la ponderación del conocimiento científico en detrimento de los saberes producidos desde la praxis por parte de los sujetos "no académicos". En ese plano, la ciencia construye su mirada analítica a partir del acto de distanciarse de la realidad para posicionarse desde "afuera del mundo", en procura de lograr neutralidad y universalidad. Así, "los demás conocimientos, desplegados históricamente por la humanidad durante milenios, son vistos como anecdóticos, superficiales, folclóricos, mitológicos, 'pre-científicos' y, en cualquier caso, como pertenecientes al pasado de Occidente" (Castro Gómez, 2007, p. 88). Como parte de esta episteme, la universidad es la institución rectora del conocimiento considerado legítimo y ejerce una vigilancia sobre sus límites. Aunque, desde las perspectivas críticas, se entiende que esto constituye un campo de disputa epistemológica y metodológica que abre la posibilidad de expandir esos límites y avanzar hacia la legitimación de otros saberes (ancestrales, colectivos y populares) en el ámbito académico. 
La coproducción de conocimiento entre investigadores y sujetos investigados, tal como lo sostienen las perspectivas aquí analizadas, supone como punto de partida una concepción del sujeto como agente con capacidad de hacer y conocer. ${ }^{8}$ Es decir, se plantea de mínima una posición de igualdad a partir del reconocimiento de la validez de los saberes que emergen de la praxis (doxa), aunque hay ciertos desplazamientos conceptuales y metodológicos respecto de cómo avanzar -sea en la línea de lo que aquí denominaremos como reivindicación, incorporación y/o producción desde esos saberes "no científicos"- en la práctica investigativa de acuerdo a cada perspectiva en particular.

En términos más específicos, la IAP postula un trabajo metodológico en el que la participación real de los sujetos investigados en el proceso de investigación va de la mano de la recuperación e incorporación de sus aportes, reflexiones e interpretaciones. Para ello, se deben generar "sesiones de retroalimentación", en donde se presenten resultados parciales y finales de la investigación (como una instancia de triangulación metodológica in situ) en pos de la creación y recreación del conocimiento. Esto abre la posibilidad tanto de generar instancias de objetivación de la realidad como de "enriquecer el conocimiento de la investigación con la participación del investigado/a, al poner a su consideración los resultados a los que se arribó, estimulando la identificación de nuevas preguntas de investigación, nuevas categorías e hipótesis" (Sirvent y Rigal, 2012, p. 74). El/la investigador/a formado/a ocupa un rol diferencial a la medida de un/a educador/a popular, en tanto selecciona, recupera, cuestiona estos aportes, y encamina un proceso grupal de producción crítica. ${ }^{9}$

En diálogo con lo anterior, la IM evidencia su posicionamiento crítico, en tanto cuestiona las dinámicas que privilegian al conocimiento considerado científico para luego ser "transferido" o "depositado" en sujetos que pasivamente reciben el producto elaborado por el académico (a la medida de la educación bancaria de Freire). En contraposición, se valoran "las dinámicas dialógicas, la centralidad de la participación, la socialización de los conocimientos y técnicas de investigación” (Jaumont y Versiani, 2016, p. 442). ${ }^{10} \mathrm{Se}$ realiza un trabajo colectivo en pos de la horizontalidad en la coproducción de conocimiento, respecto de la cual los investigadores tienen un papel específico a la hora de socializar las herramientas técnicas y teóricas acumuladas por el conocimiento científico, a la vez que se abandonan "posiciones arrogantes" que presuponen su superioridad sobre los saberes ancestrales, colectivos y populares. De a la mano de las estrategias de retroalimentación y socialización es que podemos decir que tanto la IAP como la IM buscan incorporar estos saberes en la coproducción de conocimiento.

Por otro lado, esta relación horizontal ha sido abordada a partir de ciertas conceptualizaciones en torno al "diálogo de saberes" desde las MD y la educación popular. Como parte de la crítica a la colonialidad, Castro Gómez (2007) postula una ruptura epistemológica con el pathos de la distancia, al sostener que todo/a observador/a-investigador/a es parte integral de aquello que observa/investiga, y, por consiguiente, la doxa es inherente a la producción de conocimiento. Esto debe implicar el trabajo por una universidad transcultural y transdisciplinaria, en donde puedan convivir diferentes formas de producción de conocimiento; a su vez debe evitarse por medio del diálogo de saberes que dichas formas de producción queden sometidas a la hegemonía moderna. Así, se reivindica la validez de la doxa y se pretende trabajar para que gane legitimidad al interior de las instituciones universitarias.

Por consiguiente, podemos decir que esta postura se inscribe en lo que llamamos una reivindicación de los saberes ancestrales, colectivos y populares, pero la misma no avanza en cómo incorporarlos metodológicamente en la práctica investigativa. En este sentido se expresa Michi (2020), quien sostiene que aún está pendiente la construcción de un herramental metodológico capaz de revisar críticamente las distintas racionalidades y matrices de pensamiento que entran en juego en la praxis de investigación que aboga por un diálogo de saberes. Es decir, una praxis que pueda retomar el sentido del diálogo tal como es pensado desde la educación popular freireana, a la vez que cuestionar las formas de la colonialidad muchas veces presente en el trabajo académico. Probablemente una de una de las propuestas que más tensiona este problema y cuestiona los límites epistemológicos y metodológicos del conocimiento al interior de la academia desde los ya citados enfoques situados de las MD sea la de Smith (2016), quien entiende que la descolonización de la investigación 
reside en que el conocimiento sea producido desde los saberes ancestrales, en un gesto que propone que los sujetos populares "se investiguen a sí mismos".

Por otro lado, así como en la IAP y en la IM, en la IeC encontramos, tal como la delinea Rappaport (2007), una apuesta por la incorporación de los saberes ancestrales, colectivos y populares. En este caso, se genera a partir del trabajo de coteorización entre todos aquellos que conforman el equipo de investigación, lo cual implica la "producción colectiva de vehículos conceptuales que retoman tanto un cuerpo de teorías antropológicas como a los conceptos desarrollados por nuestros interlocutores" (2007, p. 204). En términos de Katzer y Samprón (2012), hay que trascender los abordajes dicotómicos en cuanto a la relación investigador/a-sujeto investigado, entendiendo que el trabajo de campo pone en escena una relación situacional entre interlocutores distintos con capacidad de establecer y negociar acuerdos provisorios y cambiantes sobre los que se busca atender a las demandas de los sujetos de estudio como a los fines académicos. Esto indica que las estrategias metodológicas posibles en una práctica de investigación crítica se juegan en situaciones específicas, que darán mayor o menor margen para avanzar más allá del reconocimiento y reivindicación de los saberes populares hasta alcanzar su incorporación.

Por último, cabe señalar que la relación entre ciencia y otros saberes no solo se dirime en el proceso de investigación, sino también en la legitimación de los conocimientos generados. Si bien para todas las perspectivas resulta importante que el conocimiento pueda ser incorporado y legitimado al interior de la academia, para la IAP y la IM la validez del conocimiento coproducido se juega significativamente en su legitimación social; esto es, en sus aportes a los objetivos políticos del grupo en lucha. De este modo, no solo se requiere atender a las demandas de los sujetos de estudio -cuestión transversal a las perspectivas-, sino también participar en la construcción de un conocimiento necesario y pertinente para los actores sociales. Así, los saberes populares entran en el ámbito de la ciencia del mismo modo que los conocimientos científicos se validan más allá del ámbito académico.

\subsection{La relación ciencia y política}

En función a la lectura crítica propuesta es identificable un tercer eje, que atraviesa a todas las perspectivas en análisis: la relación entre lo que Leyva Solano (2018) denomina como prácticas académicas comprometidas y prácticas de activismo político (en suma, la relación entre ciencia y política). Lejos de los planteos positivistas, que abogan por una ciencia elaborada desde la neutralidad valorativa, en estas perspectivas el posicionamiento político es intrínseco a la producción de conocimiento. En la misma línea en que Elías (1990) discute la supuesta polarización entre una posición de puro compromiso o de puro distanciamiento en términos absolutos, las prácticas académicas comprometidas y las prácticas de activismo político pueden ser interpretadas como un continuo de tendencias que se superponen y tensionan de acuerdo a los procesos específicos en juego, en lo relativo al rol de los intelectuales con respecto a las luchas políticas de los sujetos de estudio.

Bajo esta idea de continuo, la IAP y la IM se destacan por un punto de partida en común, que entiende a la producción de conocimiento como una praxis, como una acción social orientada a la transformación social de la realidad y a la construcción de una sociedad más justa e igualitaria. En el marco de ambas perspectivas, el pensamiento social crítico latinoamericano propio de las décadas de 1960 y 1970 dejó una fuerte impronta sobre el significado del compromiso a partir del rol de "activistas intelectuales" (Fals Borda en Herrera Farfán y López Guzmán, 2012, p. 306), principalmente porque se entendió que la producción de conocimiento y la labor académica no son ajenas a la estructuración desigual del orden capitalista. En suma, este punto de partida supone que prácticas académicas comprometidas y prácticas de activismo político se superponen y fusionan bajo una misma praxis y horizonte.

En el caso de la IAP, esta fusión es identificable en tanto la producción de conocimiento tiene como objetivo explícito la generación de saberes emancipatorios, que resultan de procesos de enseñanza y 
aprendizaje grupales, que reúnen a todos aquellos participantes de la práctica de investigación-acción. Para ello, se generan instancias de articulación y participación real de todos los intervinientes (académicos y sujetos populares involucrados), en las que la guía y el ordenador del trabajo de investigación no responde a pautas académicas, sino que la validación de qué se investiga, para qué y cómo se responde colectivamente en función a los objetivos políticos de transformación social.

Como parte del proceso de refundación política de la IAP, encontramos una revalorización de la idea de compromiso político, que tiene mucha actualidad, principalmente a partir de la IM, que, ya desde su nombre, reivindica el rol militante del investigador/a. Los académicos asumen un posicionamiento ético político y se ubican al servicio de los intereses de los grupos en lucha. Sin embargo, la figura de investigador/a militante no solo refiere a investigadores formados, sino que los propios movimientos sociales y populares ocupan cada vez más un rol protagónico a partir de su propia praxis política, la cual requiere y produce nuevos conocimientos, que luego son transmitidos y recreados en sus procesos internos de formación (Michi, 2020).

En esta línea, Bringel y Versiani (2016b) postulan que científico y político son indisociables en aquellas investigaciones que integran en un mismo movimiento producción de conocimiento, acción política y transformación social. En este caso, no se postulan pautas de trabajo metodológico específicas como en la IAP, sino que se entiende que la IM es un espacio amplio que incluye a todas aquellas líneas de investigación que se guíen por este horizonte. Se valoriza la noción de retroalimentación entre marcos teóricos académicos y prácticas políticas de los movimientos sociales organizados, lo que genera transformaciones mutuas.

Tanto en las $\mathrm{MD}$ como en la $\mathrm{IeC}$ encontramos una manera alternativa de abordar la relación entre prácticas académicas comprometidas y prácticas de activismo político. Si bien comparten el posicionamiento inicial epistémico-político, que aboga por la producción de saberes para la emancipación frente a todas las formas de explotación y subalternización, presentan algunos desplazamientos. Con distintos términos, autores identificados con estas perspectivas desarrollan planteos sobre cómo pensar la relación entre agendas políticas y agendas académicas, que no confluyen en términos de fusión, sino de alineación.

En sus trabajos con comunidades indígenas y sobre ellas, Leyva y Speed (2018) postulan la necesidad de avanzar hacia una "investigación descolonizada activista". Se plantea un trabajo en colabor para la defensa de derechos, aunque académicos e indígenas tengan proyectos y agendas particulares. En esa clave se erige la figura del "investigador/a alineado/a" con el grupo organizado en lucha. El desplazamiento entre las posturas anteriores (IAP e IM) y esta aparece en la medida en que estar alineado implica que los académicos mantengan un espacio propio de independencia, a la vez que se superponen y comparten objetivos políticos con los sujetos de estudio. Esta ambivalencia se logra mediante el trabajo en colabor, a partir de la construcción de agendas compartidas que busquen atender a los intereses diferenciales de las partes involucradas en el trabajo de investigación. Así, mediante esta articulación desde la diferencia se definen objetivos de trabajo, se llevan adelante y se redactan los resultados en coautoría.

Esta idea de alineación supone que la relación entre investigadores y sujetos-actores (protagonistas políticos) conlleva una serie de tensiones más que coincidencias plenas. Esto puede significar un potencial para problematizar y hacer una lectura crítica en colabor de las jerarquías implicadas en los procesos de producción de conocimiento (Borsani, 2014). Así surgirán textos negociados, compartidos y consensuados como respuesta a la siguiente tensión: “¿Cómo ejercer desde los pueblos indígenas, el control requerido sobre las investigaciones efectuadas respetando al mismo tiempo la independencia, la autonomía y el pensamiento crítico que debe tener un/a investigador/a co-laborativo/a para llevar a cabo bien su tarea?” (Leyva y Speed, 2018, p. 14). En una línea similar, que entiende que el trabajo académico no siempre es coincidente con las agendas de los grupos en lucha, Haber (2014) sostiene que el vínculo entre investigadores y comunidades se construye desde relaciones sociales signadas por el compadrazgo y la amistad a partir de habitar un espacio común, más que desde declamaciones sobre compromiso social. Esto implica que, por momentos, los intereses y tiempos puedan ser compartidos, pero no son siempre coincidentes. 
Estas propuestas son emparentables con la manera en que se aborda la coteorización y articulación entre antropólogos y sus interlocutores en el trabajo de campo en la etnografía colaborativa (Katzer y Samprón, 2012; Rappaport, 2007). A partir de reuniones periódicas a lo largo del tiempo, se busca crear un plano de interacción común que incluya los objetivos para la acción política y los objetivos intelectuales de interpretar la realidad. Las teorizaciones colectivas se construyen desde las conversaciones surgidas en esa arena de interacción que interconecta las esferas de la práctica y de la analítica/reflexividad. Es en ese sentido que los objetivos de investigación no se funden con los objetivos políticos, sino que se busca crear una tercera instancia de articulación. Para ello, es fundamental colaborar con las demandas de los sujetos de estudio más allá de los fines estrictamente académicos, a partir de acuerdos intersubjetivos (siempre móviles y renovables) logrados en las instancias de trabajo de campo.

\section{REFleXiones FinALES}

En este trabajo partimos de la hipótesis interpretativa relativa a que las perspectivas metodológicas y epistemológicas críticas en Ciencias Sociales en clave latinoamericana tienen varios puntos en común, a la vez que tienen una serie de matices que se expresan en ciertas distinciones terminológicas y metodológicas que aquí buscamos precisar. Principalmente, hay una fuerte impronta de la crítica epistemológica a los cánones positivistas, que abogan por la producción de conocimiento a partir de una relación de distanciamiento que opera en el vínculo entre sujetos, saberes y política. Con ciertos deslizamientos conceptuales, las perspectivas analizadas (IAP, IM, MD y IeC) postulan que la práctica investigativa se juega en las relaciones sujeto-sujeto para la coproducción de conocimiento.

De acuerdo a la literatura específica disponible, consideramos que uno de los puntos que aún requieren mayor problematización y profundización refiere a las cuestiones metodológicas. Más aún, consideramos que lo metodológico es clave para concretizar los puntos de partida epistemológicos. En ese sentido, si bien excede los límites de este artículo, resulta central destinar estas reflexiones finales al abordaje del aspecto metodológico como condición de posibilidad para llevar adelante procesos de producción de conocimiento críticos.

La práctica de investigación científica implica la toma sistemática de decisiones para la producción de conocimiento. Desde una mirada crítica, entendemos que en estas decisiones se juegan muchos de los ejes de interés contenidos en los objetivos político-académicos de estas perspectivas, es decir, quiénes, cómo, cuándo y con qué criterios se toman las decisiones en un proceso de investigación. Allí reside la posibilidad de plantear posicionamientos políticos, establecer relaciones dialógicas con los sujetos de estudio, y ampliar los espacios de legitimación del conocimiento producido por fuera de los límites académicos.

Sin embargo, consideramos que existe una distancia entre la enunciación que plantea el uso de perspectivas críticas y los desafíos que trae aparejada su efectiva implementación. La distancia entre los lenguajes académicos y los requisitos formales e institucionales de la investigación científica, y los saberes y temporalidades populares requiere de instancias de negociación y articulación que pueden reflejar tensiones y contradicciones. A menudo, la posibilidad de avanzar en este sentido no responde a un diseño metodológico elaborado a priori, sino a la puesta en acto de un conjunto de prácticas implicadas en el proceso de investigación relacionadas al vínculo concreto con los sujetos investigados. Esto vuelve aún más relevante la necesidad de hacer ejercicios reflexivos sobre la propia práctica metodológica inscripta en epistemologías críticas.

Las precisiones conceptuales objeto del presente artículo plantean una serie de matices entre las perspectivas mencionadas que sin dudas requieren traducciones metodológicas particulares. $\mathrm{Al}$ respecto, reconociendo el consenso que establece que los criterios de validación del conocimiento coproducido no son puramente académicos, los planteos analizados van desde posturas que entienden que los objetivos académicos deben subsumirse y fusionarse con los objetivos políticos de transformación social y estar al 
servicio del grupo en lucha, mientras que otros prefieren plantear este vínculo en términos de alineación. Asimismo, las perspectivas analizadas adoptan de mínima una posición que reconoce como válidos los saberes ancestrales, populares y colectivos "no científicos". Ahora bien, este reconocimiento no resuelve los desafíos implicados en las variadas apuestas, por reivindicarlos o bien avanzar aún más hacia su incorporación, o directamente la producción desde dichos saberes. De igual modo, las implicancias metodológicas se vislumbran cuando se va más allá del común llamado de anudamiento entre ciencia y política, variando en su concepción acerca del rol de los intelectuales en las luchas de los sujetos de estudio, sea como prácticas académicas comprometidas o como prácticas de activismo político.

Lo anterior da cuenta de la necesidad de avanzar en el ejercicio de precisión conceptual a la hora de referirnos a las perspectivas metodológicas y epistemológicas críticas en el campo de las Ciencias Sociales, evitando caer en generalizaciones que borran la singularidad de los abordajes. Dicho ejercicio nos lleva a sostener que, aun dentro de lo crítico en clave latinoamericana, se encuentra abierta la discusión en torno a los modos de descolonización de los propios ámbitos de trabajo académicos en pos del desarrollo y puesta en práctica de metodologías que sostengan en igual medida los objetivos políticos y académicos para la coproducción de conocimiento.

\section{RefERENCiAS}

Abu-Lughod, L. (1990). Can There Be a Feminist Ethnography? Women and Performance, 5(1), 7-27.

Borsani, M.E. (2014). Reconstrucciones metodológicas y/o metodologías a posteriori. Astrolabio Nueva Época, 13, 146-168.

Bringel, B., y Maldonado, E. (2016). Pensamento Crítico Lat ino - Americano e Pesquisa Militante em Orlando Fals Borda: práxis, subversão e libertação. Dereito \& Praxis Rio de Janeiro, 7(13), 389-413.

Bringel, B., y Versiani, R. (2016a). Pesquisa Militante e Produção de Conhecimentos: o enquadramento de uma perspectiva. Disponible en: http://universidademovimentosociais.wordpress/artigos/.

Bringel, B., y Versiani, R. (2016b). A pesquisa militante na América Latina hoje: reflexões sobre as desigualdades e as possibilidades de produção de conhecimentos. Revista Digital de Direito Administrativo, 3(3), 474-489.

Castro-Gómez, S. (2005). La hybris del punto cero. Ciencia, raza e Ilustración en la Nueva Granada (1750-1816). Bogotá: Centro Editorial Javeriano.

Castro-Gómez, S. (2007). Descolonizar la universidad. La hybris del punto cero y el diálogo de saberes. En S. Castro-Gómez y R. Grosfoguel (eds.), El giro decolonial. Reflexiones para una diversidad epistémica más allá del capitalismo global (pp. 79-91). Siglo del Hombre: Bogotá.

De Oto, A. (2013). Usos de Fanon. Un recorrido por tres lecturas. Cuyo Anuario de Filosofía Argentina y Americana, $30(7): 35-60$.

De Oto, A. (2017). Fanon, crítica poscolonial y teoría. Notas en contextos latinoamericanos. Revista Intersticios de la politica y la cultura, 11, 5-24.

Elías, N. (1990). Compromiso y distanciamiento. Ensayos de sociología del conocimiento. Barcelona: Cultura libre.

Fajardo, F., Longa, F., y Stratta, F. (2012). Investigación y movimientos sociales. Problemas y perspectivas. Debates Insurgentes, 1(1), 99-113.

Freire, P. (2002). Pedagogía de la Esperanza. Un reencuentro con la Pedagogía del Oprimido. Buenos Aires: Siglo XXI.

Freire, P. (2006). Pedagogía del Oprimido. Buenos Aires: Siglo XXI.

Freire, P. (2011). Política y Educación. México D.F: Siglo XXI.

González Casanova, P. (2004). Las nuevas ciencias y las humanidades. De la academia a la política. México: Anthropos - IIS UNAM.

Guelman, A., y Palumbo, M. M. (2018). Pedagogias descolonizadoras. Formación en el trabajo en los movimientos populares. Buenos Aires; El Colectivo-CLACSO. 
Haber, A. (2011). Nometodología Payanesa: Notas de Metodología Indisciplinada. Revista de Antropología, 23, 9-49. Haber, A. (2014). Interculturalidad epistémica y acción política en la arqueología poscolonial. En M. Rivolta, M. Montenegro, L. Menezes Ferreira y J. Nastri (eds.), Multivocalidades y activaciones patrimoniales en arqueología: perspectivas desde Sudamérica (pp. 47-65). Ciudad Autónoma de Buenos Aires: Fundación de Historia Natural Félix de Azara.

Harding, S. (1987). The Metod Question. Hy-patia, 2(3), 19-35 [¿Existe un método feminista? Traducción de Gloria Elena Bernal]

Herrera Farfán, N. (2018). Saber colectivo y poder popular. Tentativas sobre Orlando Fals Borda. Ciudad Autónoma de Buenos Aires: El Colectivo.

Herrera Farfán, N., y López Guzmán, L. (comps.) (2012). Ciencia, compromiso y cambio social. Orlando Fals Borda. Antología. Montevideo: Lanzas y Letras-El Colectivo-Extensión Libros.

Jaumont, J., y Versiani Scott Varella, R. (2016). A Pesquisa militante na América Latina: Trajetória, caminhos e posibilidades. Direito \& Praxis, 7(13), 414-464.

Katzer, L., y Samprón, A. (2012). El trabajo de campo como proceso. La etnografía colaborativa como perspectiva analítica. Revista Latinoamericana de Metodología de Investigación Social, 2, 59-70.

Lander, E. (2000). Ciencias sociales: saberes coloniales y eurocéntrico. En E. Lander, (comp.), La colonialidad del saber: eurocentrismo y ciencias sociales. Perspectivas latinoamericanas (pp.11-40). Buenos Aires: CLACSO.

Lassiter, L. (2005). Collaborative Ethnography. Chicago: Chicago University Press.

Leyva, X. (2018). ¿Academia versus activismo? Repensarnos desde y para la práctica teórica-política. En X. Leyva Solano, et al. (eds.) Prácticas otras de conocimiento(s). Entre crisis, entre guerras (Tomo II) (pp.199-222). Ciudad Autónoma de Buenos Aires: CLACSO.

Leyva Solano, X., y Speed, S. (2018). Hacia la investigación descolonizada: nuestra experiencia de co-labor. En X. Leyva Solano, et al. (eds.) Prácticas otras de conocimiento(s). Entre crisis, entre guerras (Tomo I) (pp.451-480). Ciudad Autónoma de Buenos Aires: CLACSO.

Lugones, M. (2008). Género y descolonialidad. Buenos Aires: Ediciones del signo.

Maffia, D. (2007). Epistemología feminista: La subversión semiótica de las mujeres en la ciencia. Revista Venezolana de Estudios de la Mujer, 28(12), 63-98.

Marcus, G. (2008). El o los fines de la etnografía: del desorden de lo experimental al desorden barroco. Revista de Antropología Social, 17, 27-48.

Mendoza, B. (2010). La epistemología del sur, la colonialidad del género y el feminismo latinoamericano. En Y. Espinosa Miñoso (coord.), Aproximaciones críticas a las prácticas teórico-políticas del feminismo latinoamericano. Buenos Aires: En la frontera.

Michi, N. (2020). Reflexiones sobre prácticas de producción colectiva de conocimientos o pequeñas contribuciones a una agenda de trabajo. Investigación Militante. En P. Medina Melgarejo (coord.), Pedagogias del Sur en movimiento. Nuevos caminos en investigación (pp. 72-89). Veracruz: Universidad Veracruzana.

Mignolo, W. (2010). Desobediencia epistémica. Retórica de la Modernidad, Lógica de la Colonialidad y Gramática de la Descolonialidad. Buenos Aires: Del Signo.

Puentes, J. P. (2015). Descolonización metodológica e interculturalidad: Reflexiones desde la investigación etnográfica. Revista Latinoamericana de Metodología de las Ciencias Sociales, 5(2), 1-19.

Rappaport, J. (2007). Más allá de la escritura: La epistemología de la etnografía en colaboración. Revista Colombiana de Antropología, 43, 197-229.

Rappaport, J., y Ramos Pacho, A. (2005). Una historia colaborativa: retos para el diálogo indígena académico. En Historia Crítica (pp. 39-62). Bogotá: Universidad de los Andes.

Segato, R. (2013). La critica de la colonialidad en ocho ensayos y una antropologíapor demanda. Buenos Aires: Prometeo.

Sirvent, M. T., y Rigal, L. (2012). Investigación acción participativa. Un desafio de nuestros tiempos para la construcción de una sociedad democrática. Ecuador: Proyecto Páramo Andino. 
Sirvent, M. T., y Rigal, L. (2014). La investigación acción participativa como un modo de hacer ciencia de lo social. Decisio, 38, 7-12.

Smith, L. T. (2016). A descolonizar las metodologias. Investigación y pueblos indigenas. Santiago de Chile: LOM.

Walsh, C. (2007). ¿Son posibles unas ciencias sociales/culturales otras? Reflexiones en torno a las epistemologías coloniales. Nómadas, 26, 102-113.

\section{Notas}

1 Las reflexiones aquí compartidas se inscriben en el desarrollo del Proyecto de Reconocimiento Institucional "Perspectivas epistemológicas y metodológicas críticas en investigaciones en Ciencias Sociales" (2018-2020) radicado en la Facultad de Ciencias Sociales de la Universidad de Buenos Aires, Argentina.

2 Cabe aclarar que no desconocemos la importancia del diálogo entre pensadores críticos caribeños y las producciones latinoamericanas aquí analizadas. Principalmente, es destacable el impacto que han tenido Frantz Fanon, Aimé Cesaire, Edouard Glissant y Roberto Fernández Retamar, entre otros, para problematizar las herencias coloniales que atraviesan a nuestro continente. Un ejemplo claro de este diálogo posible lo encontramos en la producción de Alejandro de Oto (2013, 2017). Sin embargo, por razones de espacio y de focalización del análisis en América Latina, no desarrollaremos aquí las especificidades de estos aportes teórico-metodológicos.

3 Se destacan las epistemologías y metodologías feministas (Abu-Lughod, 1990; Harding, 1987; Maffia, 2007), tanto como a la educación popular (Freire, 2002, 2006, 2011), como perspectivas igualmente relevantes para abonar a las discusiones en torno a la producción crítica de conocimiento en el campo de las Ciencias Sociales y a la crítica al canon de la ciencia moderna occidental. De hecho, son fecundos los cruces entre las primeras y la teoría decolonial (Lugones, 2008; Mendoza, 2010; Segato, 2013), y, de igual modo, la educación popular es retomada por la IAP y la IM como parte de su tradición.

4 Por razones de espacio, no nos detendremos en este artículo en la contextualización histórica del pensamiento crítico latinoamericano y en la genealogía propiamente latinoamericana que se desprende de dicha contextualización. Para profundizar en este aspecto véase: Jaumont, J. y Versiani Scott Varella, R. (2016). "A Pesquisa militante na América Latina: Trajetória, caminhos e posibilidades", pp. 419-431.

5 La traducción del portugués es nuestra.

6 Si bien no es desarrollada en este trabajo, es interesante destacar otra línea de reflexión epistemológica sintetizada por Pablo González Casanova (2004) con respecto al pensamiento de la complejidad y a las "nuevas ciencias". Como parte de una reformulación de la relación entre sujeto y objeto, entiende que los sujetos cognitivos se relacionan entre sí y se redefinen en función a la posición que ocupan en los sistemas complejos. Esto les permite construir "verdades situadas que se adquieren a partir de una 'posición' de observación-acción" (2004, p. 107), en oposición a lo que llama la metafísica del orden o del azar absolutos, que resultan fútiles para la construcción de explicaciones sobre los fenómenos naturales y sociales. Esta propuesta revaloriza la interdisciplinariedad para el análisis de sistemas complejos que incluyen vectores físicos, biológicos, químicos, sociológicos, históricos, etc., y aboga por la reapropiación desde perspectivas críticas de las tecnologías de la información y comunicación, y de los sistemas de la complejidad en función a fines humanitarios y emancipatorios.

7 La traducción del portugués es nuestra.

8 Un punto común a las perspectivas revisitadas es la concepción de los sujetos involucrados en la producción de conocimiento en su doble condición de mente y cuerpo. El cuerpo se encuentra presente en las posibilidades y modos de participación de los sujetos en el quehacer investigativo; en la comprensión de los conocimientos como biográficamente situados, lo que permite validar los saberes populares como una forma particular de aproximarse a la realidad; en las implicaciones de los/as investigadores/as al vincularse comprometidamente con sus sujetos de estudio. La alusión a la corporalidad se encuentra particularmente enfatizada en las $\mathrm{MD}$ a través de conceptos como geopolítica y corpopolítica del conocimiento (Mignolo, 2010). Así, las MD discuten con los postulados que sostienen que el conocimiento es resultado de una elaboración abstracta, de un sujeto investigador situado en no-lugares y no-tiempos; es decir, un conocimiento descorporizado y deslocalizado, que supone un borramiento de la relación entre sujeto, conocimiento producido y relaciones de dominación/explotación/sujeción que atraviesan esa producción (Castro-Gómez, 2005; Mignolo, 2010).

9 La noción de devolución se encuentra ya presente en la obra de Fals Borda, quien postulaba la importancia de una devolución sistemática y por niveles, como parte de la vinculación con los sujetos en estudio. Si bien Sirvent y Rigal (2012) aclaran explícitamente que "no es una mera devolución de los resultados parciales o finales de la investigación" (p. 73), existe un amplio debate que cruza las prácticas investigativas y las reflexiones sobre las mismas en torno a la idea de devolución, en cuanto a que continúa enfatizando la agencia del investigador/a.

10 La traducción del portugués es nuestra. 